\title{
Chronic Diclofenac Exposure Increases Mitochondrial Oxidative Stress, Inflammatory Mediators, and Cardiac Dysfunction
}

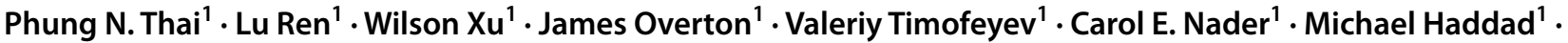 \\ Jun Yang ${ }^{2}$ - Aldrin V Gomes ${ }^{3}$. Bruce D. Hammock ${ }^{2} \cdot$ Nipavan Chiamvimonvat $^{1,4,5} \cdot$ Padmini Sirish $^{1,5}$ (1)
}

Accepted: 27 August 2021 / Published online: 9 September 2021

(c) The Author(s) 2021

\begin{abstract}
Purpose Nonsteroidalanti-inflammatory drugs (NSAIDs) are among one of the most commonly prescribed medications for pain and inflammation. Diclofenac (DIC) is a commonly prescribed NSAID that is known to increase the risk of cardiovascular diseases. However, the mechanisms underlying its cardiotoxic effects remain largely unknown. In this study, we tested the hypothesis that chronic exposure to DIC increases oxidative stress, which ultimately impairs cardiovascular function.

Methods and Results Mice were treated with DIC for 4 weeks and subsequently subjected to in vivo and in vitro functional assessments. Chronic DIC exposure resulted in not only systolic but also diastolic dysfunction. DIC treatment, however, did not alter blood pressure or electrocardiographic recordings. Importantly, treatment with DIC significantly increased inflammatory cytokines and chemokines as well as cardiac fibroblast activation and proliferation. There was increased reactive oxygen species (ROS) production in cardiomyocytes from DIC-treated mice, which may contribute to the more depolarized mitochondrial membrane potential and reduced energy production, leading to a significant decrease in sarcoplasmic reticulum (SR) $\mathrm{Ca}^{2+}$ load, $\mathrm{Ca}^{2+}$ transients, and sarcomere shortening. Using unbiased metabolomic analyses, we demonstrated significant alterations in oxylipin profiles towards inflammatory features in chronic DIC treatment.

Conclusions Together, chronic treatment with DIC resulted in severe cardiotoxicity, which was mediated, in part, by an increase in mitochondrial oxidative stress.
\end{abstract}

Keywords Mitochondria $\cdot$ Oxidative stress $\cdot$ Nonsteroidal anti-inflammatory drug $\cdot$ Diclofenac

Nipavan Chiamvimonvat

nchiamvimonvat@ucdavis.edu

$\triangle$ Padmini Sirish

psirish@ucdavis.edu

1 Department of Internal Medicine, Division of Cardiovascular Medicine, University of California, Davis, 451 Health Science Drive, CA 95616 Davis, USA

2 Department of Entomology and Nematology and Comprehensive Cancer Center, University of California, Davis, CA, USA

3 Department of Physiology and Membrane Biology, University of California, Davis, CA, USA

4 Department of Pharmacology, University of California, Davis, CA, USA

5 Department of Veterans Affairs, Northern California Health Care System, 10535 Hospital Way, Mather, CA 95655, USA

\section{Introduction}

Nonsteroidal anti-inflammatory drugs (NSAIDs) are widely available over the counter and commonly prescribed in the USA [1]. Although they have a relatively good safety profile, their widespread availability, uncontrolled disposal, and improper usage can be detrimental to the environment and human health. Indeed, improper disposal of these drugs leads to their accumulation in the water system [2], that are harmful to human health [3]. Additionally, since NSAIDs are so common and exist in many varieties that are often prescribed with other drugs, it is easy to unknowingly exceed the recommended dosage [4]. One of the known adverse effects of taking NSAIDs is elevated risks for cardiovascular complications, such as elevated blood pressure and myocardial infarction [5]. However, the exact mechanism underlying the increased susceptibility to these cardiovascular events is not completely understood. 
One of the critical signaling pathways activated during inflammation is the arachidonic acid (ARA) pathway. NSAIDs exert their inflammatory actions by targeting the enzymes cyclooxygenase (COX)- 1 and COX- 2 in the ARA metabolic pathway. Although NSAIDs predominantly induce their effects on these enzymes, they do so with varying degrees [6]. The actions of COX-1 and COX-2 on ARAs result in the production of prostaglandins and thromboxane including prostaglandin $\mathrm{E}_{2}\left(\mathrm{PGE}_{2}\right)$, prostaglandin $\mathrm{D}_{2}$ $\left(\mathrm{PGD}_{2}\right)$, prostaglandin $\mathrm{F}_{2 \alpha}\left(\mathrm{PGF}_{2 \alpha}\right)$, prostacyclin $\left(\mathrm{PGI}_{2}\right)$, thromboxane (TXA2), and related compounds that contribute to the inflammatory response [7]. Indeed, the differential level of inhibition as well as differential inhibition of COX-1 and COX-2 may produce varying toxicity [8]. Additionally, with the widespread availability of NSAIDs, their common usage, and improper disposal, the cumulative, inhibitory effects of the COX enzymes can result in profound cardiovascular consequences.

Recent emerging evidence has suggested that inhibition of the COX enzymes induces oxidative stress [9]. The mitochondria are the primary site for reactive oxygen species (ROS) production through their role in energy generation. This energy generation, although efficient, is prone to produce ROS, which normally are scavenged and detoxified by the cellular antioxidant system [10]. Under conditions of increased chronic oxidative stress, however, ROS generation can be overwhelming and detrimental to cellular function and may induce apoptosis [11]. Previous studies have demonstrated that ROS can directly increase the production of extracellular matrix as well as mitochondrial structural and membrane potential damage, leading to cardiac fibrosis [12]. Since the heart is an energetically demanding organ, it is comprised of a dense network of mitochondria. It is estimated that about $25-30 \%$ of the cardiomyocytes volume is occupied by mitochondria [13] and the heart is particularly susceptible to damage through oxidative stress, which can result in a plethora of cardiovascular complications including cardiac fibrosis and dysfunction [14]. It is well documented that the risk for cardiovascular events increases with the usage of NSAIDs [15]; however, it remains unclear what are the underlying mechanisms.

A recent in vitro study involving exposure of immortalized human cardiomyocytes to diclofenac (DIC) demonstrated an increase in ROS production, a decrease in mitochondrial membrane potential, and cardiotoxicity leading to cell death [16]. In our study, we tested the hypothesis that chronic treatment with a commonly prescribed NSAID, DIC, increases oxidative stress, which ultimately impairs cardiac function in vivo. Chronic treatment with DIC results in significant systolic and diastolic dysfunction as assessed by in vivo echocardiography with a marked decrease in single-cell shortening and $\mathrm{Ca}^{2+}$ transients. Metabolomic profiling, single-cell flow cytometric analyses, and mitochondrial function were performed to determine the underlying mechanisms of cardiac dysfunction.

\section{Materials and Methods}

\section{Echocardiography}

Eight to 12-week-old male and female C57BL/6 mice were treated with DIC $(15 \mathrm{mg} / \mathrm{kg} /$ day $)$ or vehicle alone in drinking water for 4 weeks based on our previous studies and subsequently monitored in accordance with approved protocols of the IACUC Committee at the University of California, Davis. Systolic function (M-mode images) was measured in conscious mice and diastolic function (pulse-wave Doppler and tissue Doppler) was measured in the presence of $0.5-1 \%$ isoflurane.

\section{Blood Pressure Recordings}

Mice were acclimated to the non-invasive, tail vein blood pressure recording system (Kent Scientific Corporation) for 5 days before actual measurements were taken.

\section{Mitochondrial Membrane Potential}

Mitochondrial membrane potential was assessed in freshly isolated control and DIC-treated cardiomyocytes using tetramethylrodamine ester (TMRM) [17]. Cardiomyocytes were incubated with TMRM and the recordings analyzed using ImageJ FIJI Software.

\section{Mitochondrial $\mathrm{Ca}^{2+}$ Uptake}

Mitochondrial $\mathrm{Ca}^{2+}$ uptake was monitored in freshly isolated control and DIC-treated cardiomyocytes using X-Rhod-1 AM (ThermoFisher Scientific) [17]. Cells were loaded with X-Rhod-1 AM for 40 min at $37{ }^{\circ} \mathrm{C}$. Mitochondrial $\mathrm{Ca}^{2+}$ uptake was monitored by the change in fluorescence intensity, normalized to the baseline fluorescence intensity, after the addition of $5 \mu \mathrm{M} \mathrm{Ca}^{2+}$ and $10 \mu \mathrm{M} \mathrm{Ca}^{2+}$.

\section{ROS Production}

Superoxide generation was monitored in freshly isolated control and DIC-treated cardiomyocytes using MitoSox Red (ThermoFisher Scientific) [17]. Intact cells were loaded with MitoSox Red for $30 \mathrm{~min}$ at $37{ }^{\circ} \mathrm{C}$. ROS generation was monitored after field stimulation $(0.5 \mathrm{~Hz}$ and $4 \mathrm{~Hz})$ and application of $100 \mathrm{nM}$ isoproterenol in intracellular solution. 


\section{Cardiomyocyte Shortening, $\mathrm{Ca}^{2+}$ Transient (CaT), and Sarcoplasmic Reticulum (SR) Load}

Cell shortening, CaT, and SR load were detected using IonOptix system [18] from freshly isolated cardiomyocytes from control and DIC-treated mice. Cell contraction was measured using a high-speed video camera to record sarcomere movement. The sarcomere was used to calculate the sarcomere length using the FFT algorithm. For CaT and SR load experiments, cells were loaded with Fluo- 4 and paced at $10 \mathrm{~V}$ at a frequency of $0.5 \mathrm{~Hz}$. To induce maximum sarcoplasmic reticulum, $20 \mathrm{mM}$ caffeine was applied.

\section{Flow Cytometry Analyses of Cardiac Fibroblasts and Cardiomyocytes}

Isolated cardiac cells from control and DIC-treated mice were filtered through $200-\mu \mathrm{m}$ cell strainer, re-suspended in $\mathrm{Ca}^{2+}$ and $\mathrm{Mg}^{2+}$ free phosphate-buffered saline (PBS), fixed with $0.4 \%$ paraformaldehyde, before treating with antibodies.

\section{Plasma Cytokine Levels}

Plasma cytokine levels from samples collected 4 weeks after DIC treatment were analyzed using a Cytometric Bead Array kit and FCAP Array software following the manufacturer's protocol [19].

\section{Metabolomic Profiling of Oxylipins}

Oxylipin profiling was performed using a modification of a previously published method $[19,20]$.

\section{Statistical Analysis}

All data are reported as mean \pm standard error, unless otherwise stated. Statistical comparisons were analyzed by Student's $t$-tests or one-way ANOVA followed by Bonferroni tests for post hoc comparison using GraphPad Prism version 9 (San Diego, CA). Multivariate statistical analysis was performed using MetaboAnalyst version 5.0 to analyze the distinct clustering among groups. Oxylipins were represented on a heat map using Euclidean distance and Ward's clustering. Unsupervised principal component analysis (PCA) was performed to identify possible trends. Partial least-squares discriminant analysis (PLS-DA) was applied to test the differences between control and DIC-treated groups. Validation of the model was performed using $R^{2}$ and $Q^{2}$ values derived from cross-validation algorithm. Statistical significance was considered to be achieved when $P<0.05$.

\section{Results}

\section{Chronic DIC-Treated Mice Exhibited Impaired Cardiac Function}

Recent evidence has suggested that inhibition of COX induces oxidative stress [9]. Since the heart relies on a dense network of mitochondria to supply energy, elevated oxidative stress is especially detrimental to the constantly beating heart. To investigate whether chronic DIC treatment affects cardiovascular function, mice were treated with $(15 \mathrm{mg} /$ $\mathrm{kg} / \mathrm{day}$ ) DIC for 4 weeks and subsequently monitored. The dosage was selected based on prior studies [21, 22]. Whole heart images showed no evidence of cardiac hypertrophy or chamber dilatation with no significant differences in the heart weight/body weight ratios between chronic exposure group and control (Figures S1A-D). However, there was a significant increase in mortality in the DIC-treated group (Figure S1E). Of those mice that survived, echocardiographic measurements revealed severely depressed cardiac function. Representative M-mode tracing at the parasternal short axis is shown for the two groups (Fig. 1a). Recordings were performed in conscious mice to eliminate the effects of volatile anesthetic [23]. There were no significant changes in heart rate in the chronic exposure group (Fig. 1b); however, left ventricular mass was significantly higher in DIC-treated mice, with a concomitant decrease in fractional shortening and ejection fraction (Fig. 1c-e). Diastolic function was also assessed by monitoring blood flow velocity through the mitral valve (MV) during early (E wave) and late diastole (A wave) as shown in the representative images in Fig. 1f. With treatment, there was a decline in the MV E/A ratio (Fig. 1g) with a prolongation of the E-wave deceleration time (Fig. 1h) and isovolumetric relaxation time (Fig. 1i). Together, these data suggest that chronic DIC treatment impaired both systolic and diastolic function.

Additionally, NSAIDs have been shown to elevate blood pressure [24] and increase the risk of cardiac arrhythmias $[3,25]$. We measured systolic and diastolic blood pressure using tail cuffs. Mice were acclimated with the procedure 5 days prior to blood pressure recordings. Training was done using positive reinforcement. Under our treatment regimen, chronic treatment did not affect systolic and diastolic blood pressure (Fig. 1j). In addition, we recorded ECGs in anesthetized mice after 4 weeks of treatment. Representative ECG traces are shown in Fig. 1k. There were no significant differences in RR, PR, and $\mathrm{QT}_{\mathrm{c}}$ intervals (Fig. 11). Taken together, our data suggest that chronic exposure to DIC depressed 


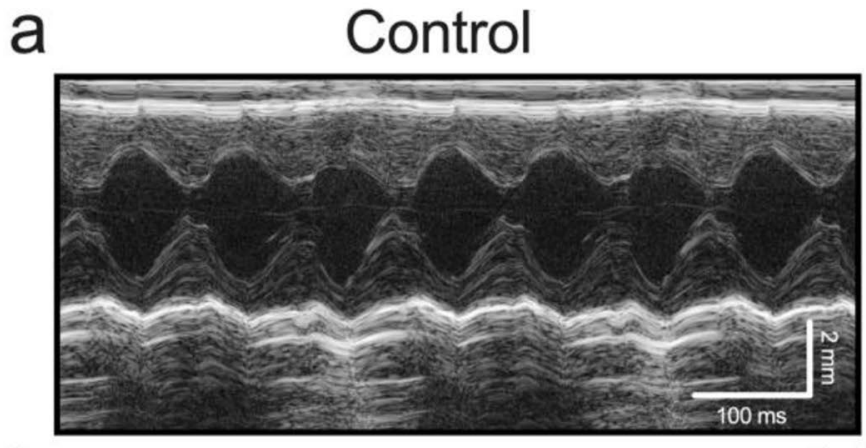

Diclofenac
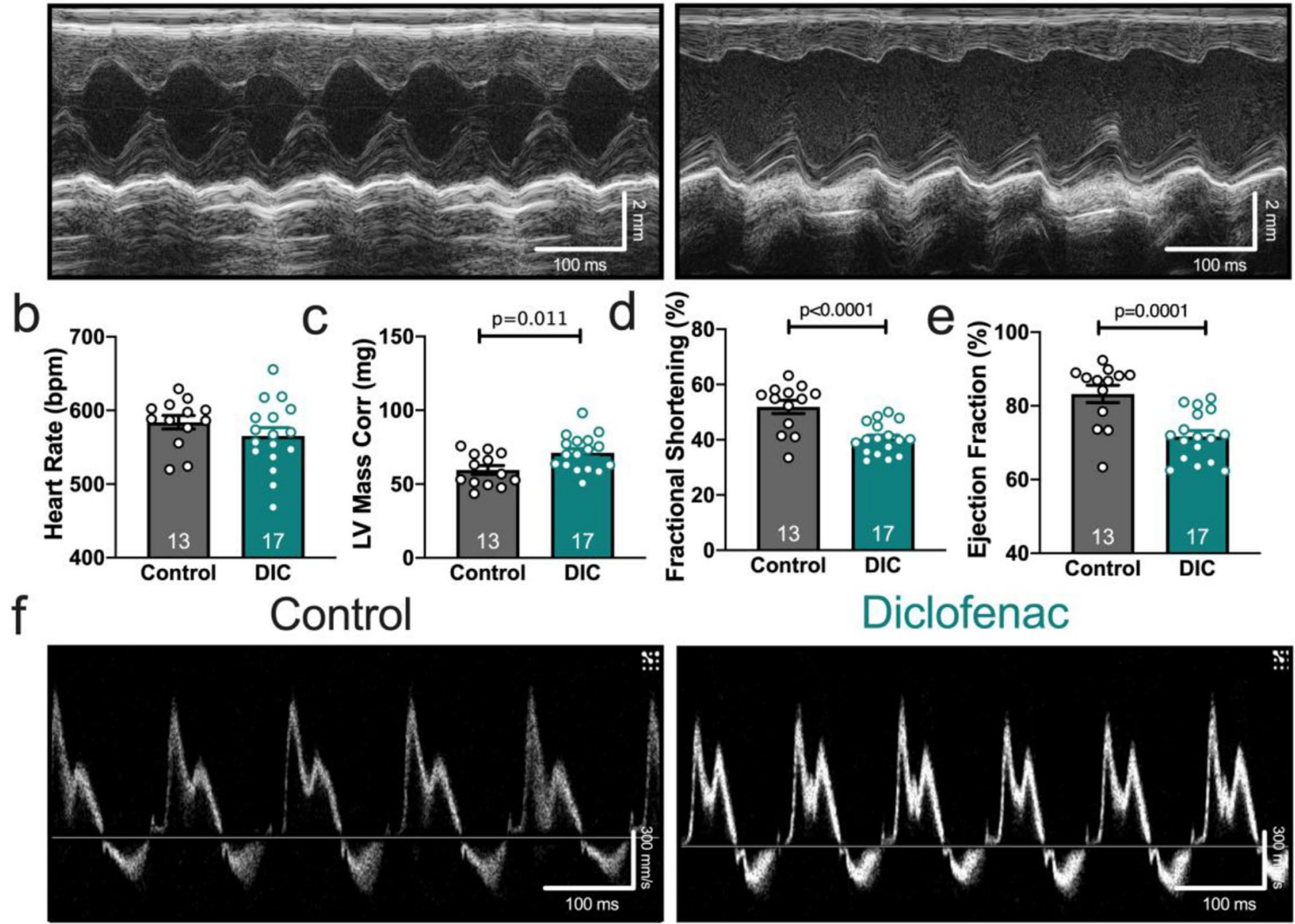

g
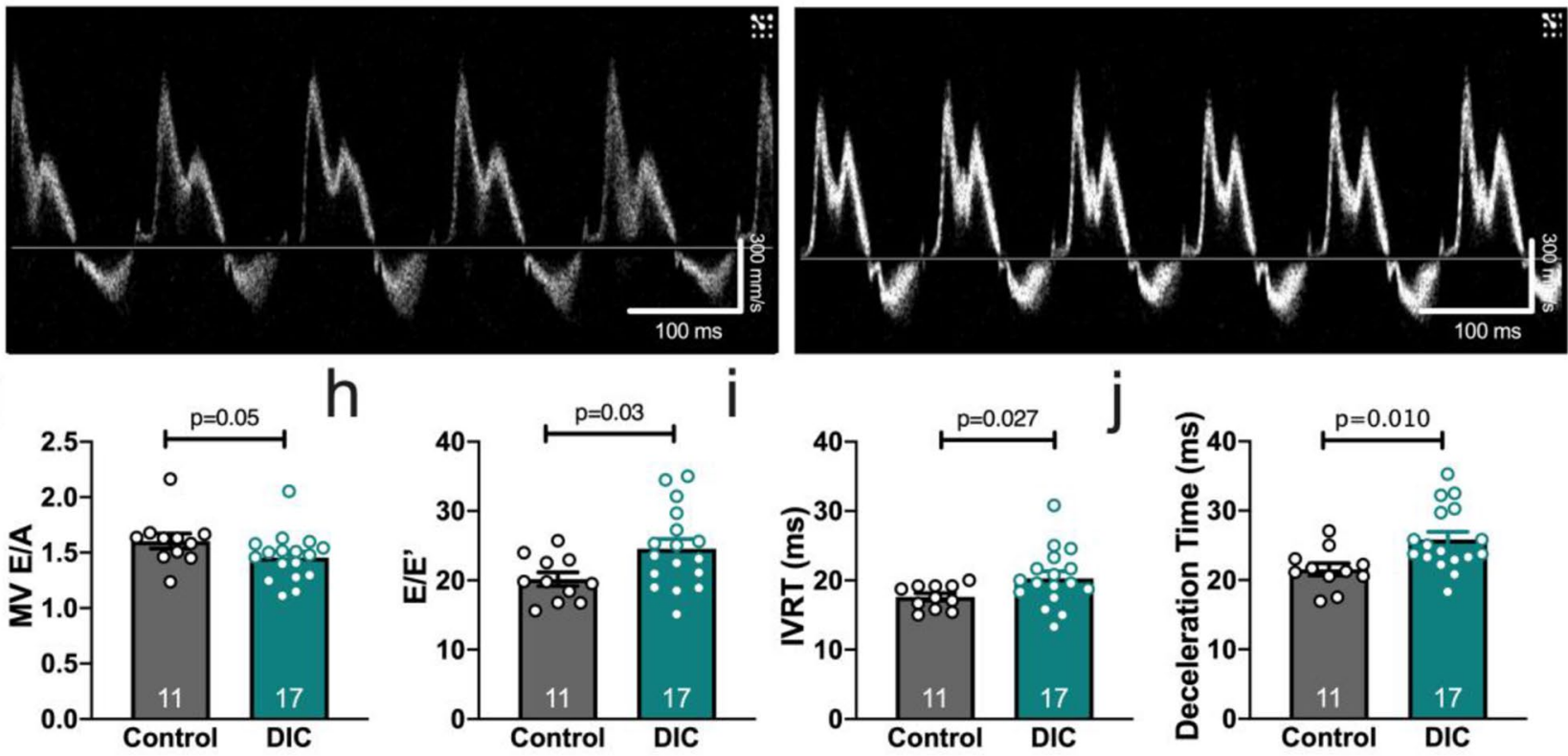

k

Control
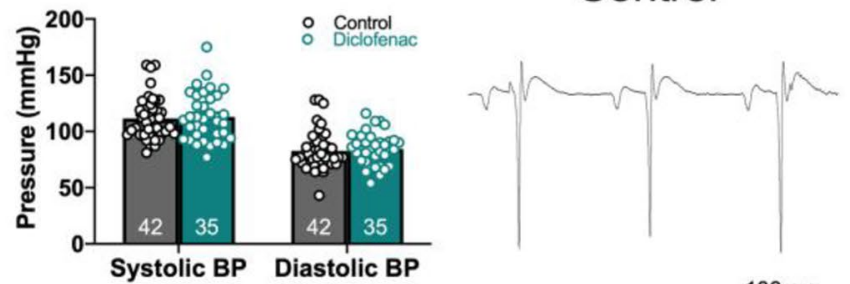

$100 \mathrm{~ms}$

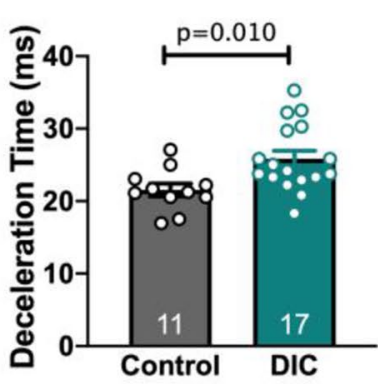

Diclofenac $\mathrm{m}$

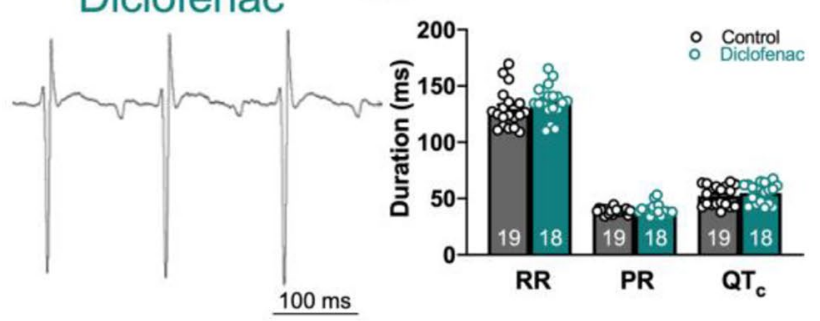


४Fig. 1 DIC-treated mice exhibited impaired cardiac function. After 4 weeks, mice were subjected to cardiovascular analyses. a Representative M-mode images at the parasternal short axis are shown. b Heart rate did not vary between the two groups in conscious echocardiography recordings. c Structurally, left ventricular mass (corrected) was significantly higher in DIC-treated mice. d Additionally, DIC-treated mice exhibited depressed systolic function, as evident by the reduced fractional shortening and e ejection fraction. $\mathbf{f}$ Blood flow velocity through the mitral valve was utilized to assess diastolic function as shown in the representative images. $\mathrm{g}$ With DIC treatment, the mitral valve E/A ratio was significantly lower. $\mathbf{h}$ The E/E' ratio, $\mathbf{i}$ isovolumentric relaxation time (IVRT), and $\mathbf{j}$ deceleration time were significantly prolonged. $\mathbf{k}$ Blood pressure, as measured by tail cuff pressure, was not altered with DIC treatment. 1 Electrocardiography recordings, as seen in the representative images, and $\mathbf{m}$ quantitatively with analyses of RR, PR, and $\mathrm{QT}_{c}$, did not show any difference between DIC-treated mice and control mice. Data represented as mean \pm SEM. Statistical significance was considered to be achieved when $P<0.05$ by $t$-test

cardiac contractile function, but did not affect systemic blood pressure or heart rate or rhythm.

\section{Mechanistic Underpinnings of the Observed Systolic and Diastolic Dysfunction in Chronic DIC Exposure Mice, Revealed Using Single-Cell Shortening and $\mathrm{Ca}^{2+}$ Transients Analyses}

To determine the mechanisms underlying the observed systolic and diastolic dysfunction in the chronic DIC-treated group, we took advantage of single-cell analyses to quantify sarcomere shortening and $\mathrm{Ca}^{2+}$ dynamics using IonOptix system. In corroboration with our echocardiography data, cardiomyocytes isolated from DIC-treated mice exhibited reduced cell shortening as shown in Fig. 2a. Quantitatively, percentages of cell shortening were significantly decreased with treatment (Fig. 2b, $p<0.0001$ ). Moreover, the time to reach $50 \%$ of the peak amplitude (Fig. 2c) and the decay time to $50 \%, 70 \%$, and $90 \%$ from peak amplitude were significantly prolonged in treated cardiomyocytes (Fig. 2d).

Since $\mathrm{Ca}^{2+}$ plays a pivotal role in mediating cellular contractility, we examined $\mathrm{Ca}^{2+}$ transients and the sarcoplasmic reticulum (SR) $\mathrm{Ca}^{2+}$ load. Representative traces of $\mathrm{Ca}^{2+}$ transient recordings are shown in Fig. 2e. There was a significant decrease in the peak amplitude of the $\mathrm{Ca}^{2+}$ transients in DIC-treated cardiomyocytes (Fig. 2f). Although there were no changes in the time-to-peak amplitude in treated cardiomyocytes (Fig. $2 \mathrm{~g}$ ), the decay time was significantly prolonged in the treated cardiomyocytes (Fig. 2h). To acquire the SR $\mathrm{Ca}^{2+}$ load, we applied caffeine to induce maximal release of $\mathrm{Ca}^{2+}$ from the SR, as shown by the representative traces in Fig. $2 \mathrm{i}-\mathrm{j}$. Chronic DIC treatment significantly decreased the SR Ca ${ }^{2+}$ load (Fig. 2j). However, there were no changes in the kinetics in the presence of caffeine (Fig. 2k-l), suggesting no significant changes in the $\mathrm{Na}^{+}-\mathrm{Ca}^{2+}$ exchange activities. Therefore, the significant decrease in $\mathrm{SR} \mathrm{Ca}^{2+}$ load is likely a result of a decrease in SR $\mathrm{Ca}^{2+}$-ATPase (SERCA) activities, a well-described remodeling of $\mathrm{Ca}^{2+}$ cycling proteins in cardiac hypertrophy and failure [26]. Together, our data demonstrate that chronic DIC treatment significantly reduced $\mathrm{SR} \mathrm{Ca}^{2+}$ load, leading to a reduction in $\mathrm{Ca}^{2+}$ transients and single-cell shortening as well as prolongation of the decay kinetics of both sarcomere shortening and $\mathrm{Ca}^{2+}$ transients (Fig. $2 \mathrm{c}$ and h). The net effects are systolic and diastolic dysfunction as we have demonstrated at the in vivo level.

\section{Chronic DIC Treatment Increased Cardiac Fibroblast Proliferation}

Since DIC has been associated with increase cardiovascular risk [3, 27], and we found that DIC treatment resulted in impaired systolic and diastolic cardiac function, we investigated structural changes in the heart. Cardiac fibroblasts are the most important contributors of the collagen matrix deposition. To evaluate structural changes, cardiac sections from control and DIC-treated mice were stained with Masson's Trichrome (Figure S1F). Since collagen staining was not evident in the cardiac sections, increase in cardiac fibroblasts was quantified using flow cytometry from isolated cardiac cells. We have previously characterized cardiac fibroblasts as cells expressing Thy $1.2^{+} / \mathrm{Lin}^{-} / \mathrm{CD} 45^{-} / \mathrm{CD} 31^{-}$using flow cytometry [19]. When digested single cells from control and DIC-treated mice were analyzed by flow cytometry, we saw a significant increase in percentages of fibroblasts in the DIC-treated group compared to the control (Control $36 \pm 1 \%$, DIC $42 \pm 1 \%$, Fig. 3a-b). We further utilized Ki67 to analyze the proliferative capacity among cardiac fibroblasts with DIC treatment. There was a significant increase in the percentage of Ki67 in the DIC-treated fibroblasts compared to control (Control $2 \pm 0.5 \%$, DIC $6 \pm 1 \%$, Fig. $3 c-d$ ).

\section{Chronic DIC Treatment Increased Oxidative Stress in Fibroblasts and Cardiomyocytes}

Exposure to DIC has been shown to increase oxidative stress. Increased levels of ROS lead to dysfunction of multiple biological signaling pathways[16, 21]. Therefore, we quantified the level of ROS in cardiac cells, specifically cardiomyocytes and fibroblasts. Analysis of cardiomyocytes from the DIC-treated and control groups revealed a significant increase in ROS in DIC-treated cardiomyocytes compared to control cardiomyocytes (Fig. 3e-f). We also demonstrate a significant increase in ROS in DIC-treated fibroblasts compared to control fibroblasts (Fig. 3g-h).

Increase in ROS has been shown to activate members of the mitogen-activated protein kinase (MAPK) signaling cascade [21]. Therefore, we investigated the activation of downstream members of the MAPK cascade, the 


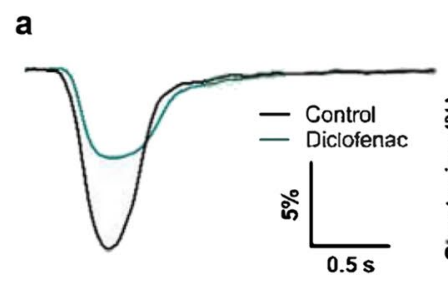

e
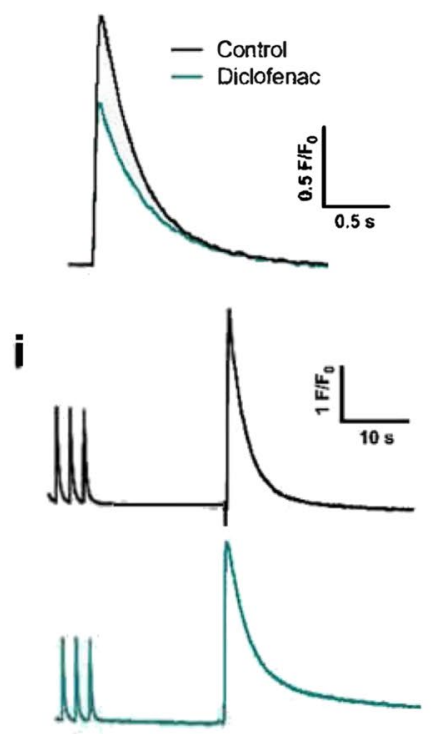

b

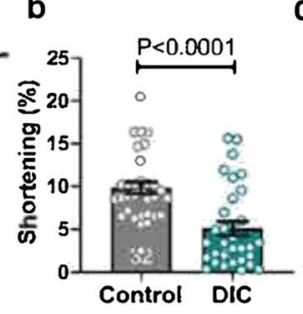

f
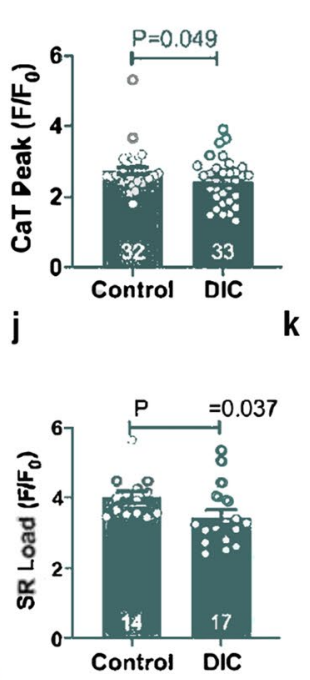

C

9
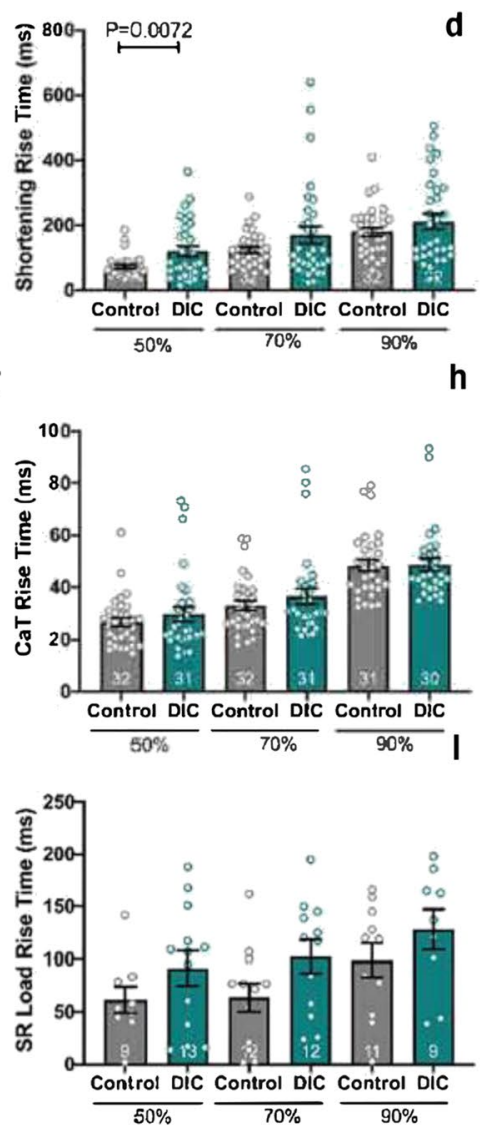

h
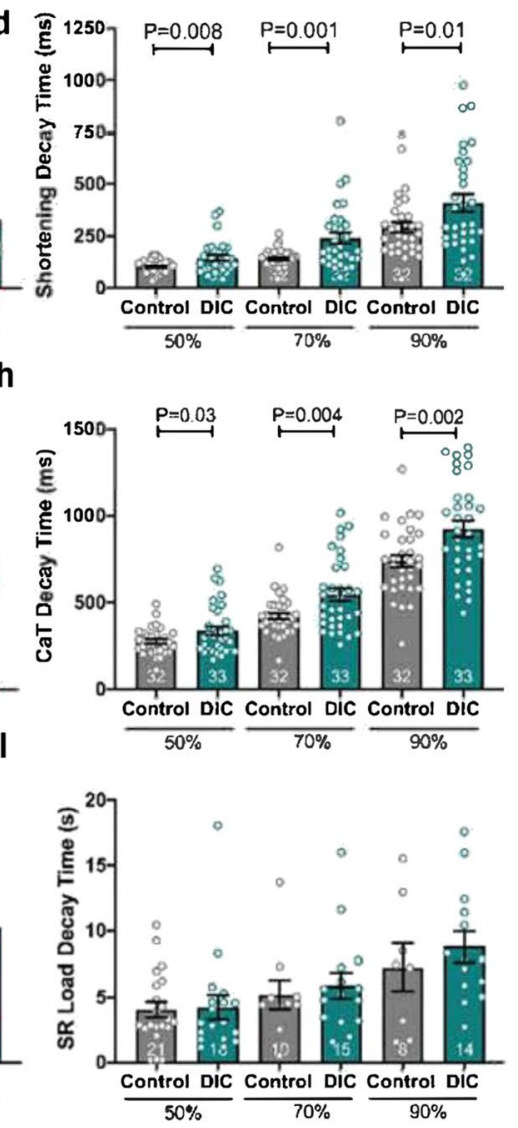

Fig. 2 DIC treatment reduced sarcomere shortening, $\mathrm{Ca}^{2+}$ transient, and SR load in isolated cardiomyocytes. Cardiomyocytes were isolated from both groups and loaded with Fluo-4 to monitor $\mathrm{Ca}^{2+}$ dynamics. a Cardiomyocyte shortening was significantly impaired with DIC treatment, as manifested in the representative traces. b The percentage of shortening was reduced, $\mathbf{c}$ while the rise time at $50 \% \mathrm{~d}$ and shortening decay time at $50 \%, 70 \%$, and $90 \%$ were prolonged. e Representative traces of $\mathrm{Ca}^{2+}$ transients are shown for both groups. $\mathbf{f}$ With treatment, there was a decrease in peak $\mathrm{Ca}^{2+}$ transient amplitude. g Although the time it took to reach the peak was not different, $\mathbf{h}$ there was a significant prolongation of the $\mathrm{Ca}^{2+}$ transient decay time at $50 \%, 70 \%$, and $90 \%$. i Additionally, the sarcoplasmic reticulum load was decreased, as shown in the representative images and $\mathbf{j}$ a significant decrease in SR $\mathrm{Ca}^{2+}$ load in the chronic exposure group. $\mathbf{k}$ and $\mathbf{l}$ There was no significant differences in $\mathrm{SR} \mathrm{Ca}^{2+}$ load rise time and the decay time between the two groups. Data expressed as mean \pm SEM. $n=5-6$ mice for each group. Statistical significance was considered to be achieved when $P<0.05$ by $t$-test extracellular signal-regulated kinase 1 and $2($ ERK1/2) in fibroblasts and cardiomyocytes. Here, there was a significant increase in pERK1/2 in DIC-treated cardiomyocytes (Fig. 3i-j). Similarly, there was a significant elevation in the levels of phosphorylated ERK1/2 (pERK1/2) in fibroblasts (Control $5 \pm 0.5 \%$ vs. DIC $10 \pm 1 \%$, Fig. $3 \mathrm{k}-1$ ).

\section{Chronic DIC Treatment Impaired Mitochondrial Function}

As an energetically demanding organ, the heart is densely packed with mitochondria. Proper communication with these mitochondria is crucial for sufficient energy production on a beat-to-beat basis [28]. To determine the effects of DIC treatment on mitochondrial function, we examined mitochondrial membrane potential, $\mathrm{Ca}^{2+}$ uptake, ROS production, and ATP level. We demonstrate that with DIC treatment, mitochondrial membrane potential was significantly depolarized (Fig. 4a). Additionally, permeabilized DIC-treated cardiomoycytes exhibited reduced mitochondrial $\mathrm{Ca}^{2+}$ uptake when challenged with 5 and $10 \mu \mathrm{M}$ of $\mathrm{Ca}^{2+}$, as shown in the representative traces and quantitatively (Fig. $4 \mathrm{~b}-\mathrm{c}$ ). Since ROS is known to increase after $\beta$-adrenergic receptor stimulation and cellular stress, [29] we subjected cardiomyocytes to both isoproterenol (ISO) and field stimulation (FS). With $100 \mathrm{nM}$ ISO and $0.5 \mathrm{~Hz}$ FS, we observed a significant increase in ROS in chronic DIC-treated cardiomyocytes relative to control, as depicted in Fig. 4d. Even with an increase in FS to $2 \mathrm{~Hz}$, treated cardiomoycytes still exhibited higher ROS generation relative to control (Fig. 4e). Since reduced mitochondrial $\mathrm{Ca}^{2+}$ uptake and elevated ROS generation can impair energy production, [30] we directly monitored the ATP level after Complex I ( $5 \mathrm{mM}$ pyruvate $/ 5 \mathrm{mM}$ malate) and Complex II ( $5 \mathrm{mM}$ succinate) mediated respiration. With Complex I substrates, the 


\section{Cardiac Fibroblasts}
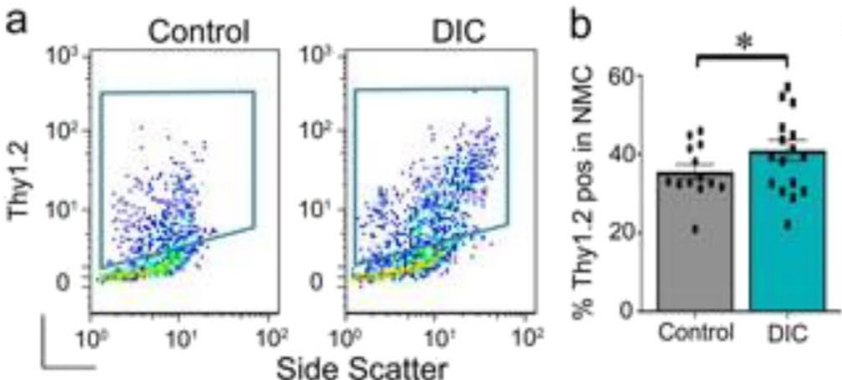

ROS in Cardiomyocytes
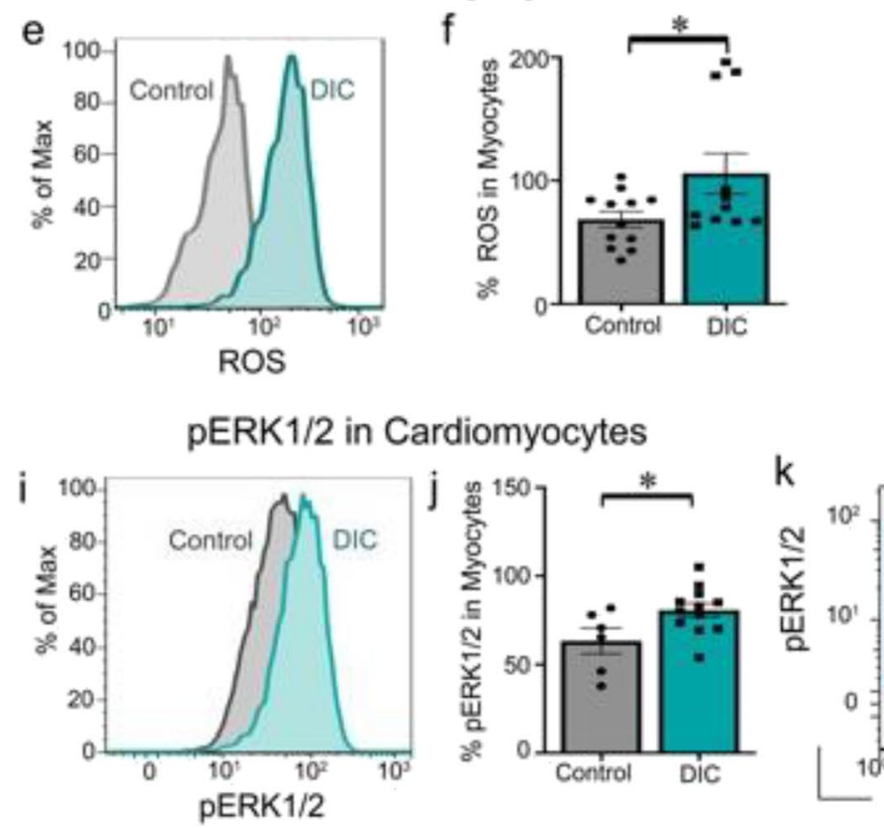

Fig. 3 DIC treatment increases cardiac fibroblast proliferation and oxidative stress. a Flow cytometric analysis of the increase percentage of cardiac fibroblasts in the DIC-treated mice compared to control mice. b Summary data from a. $n=13-18$ mice per group. c Flow cytometric analysis showing the increase proliferation of cardiac fibroblasts with DIC treatment. d Summary data from c. $n=9-14$ mice per group. e and $\mathbf{f}$ Increased oxidative stress in cardiomyocytes

ATP generation was significantly lower in the treated cardiomyocytes (Fig. 4f). This was also seen after using Complex II substrate. Together, these data suggest that mitochondrial function was significantly impaired, at least in part, due to a significant increase in ROS generation, that may further contribute to an increase in mitochondrial oxidative stress.

\section{Chronic DIC Treatment Alters the Oxylipin Profile and Increases Inflammatory Cytokines}

Polyunsaturated fatty acids including ARA, eicosapentaenoic acid (EPA), docosahexaenoic acid (DHA), linoleic acid (LA), and $\alpha$-linolenic acid (ALA) can be metabolized via

\section{Ki67 in Fibroblasts}
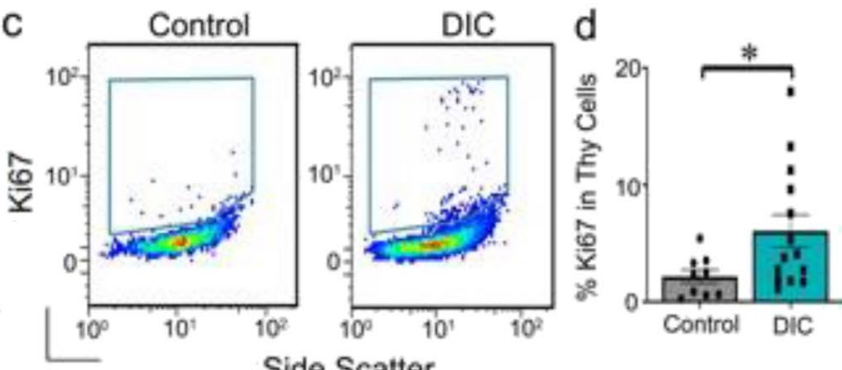

Side Scatter

ROS in Fibroblasts

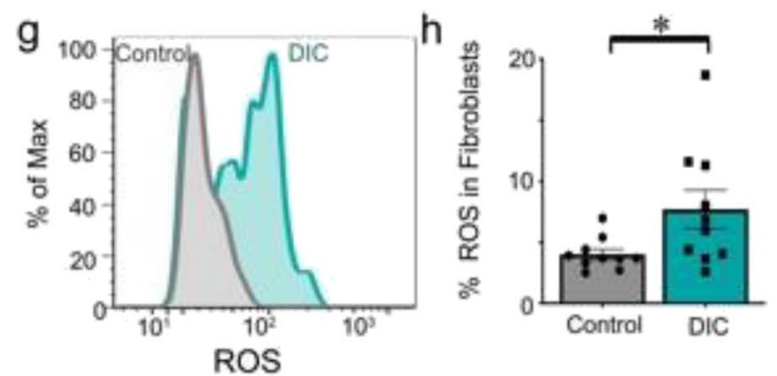

pERK1/2 in Fibroblasts
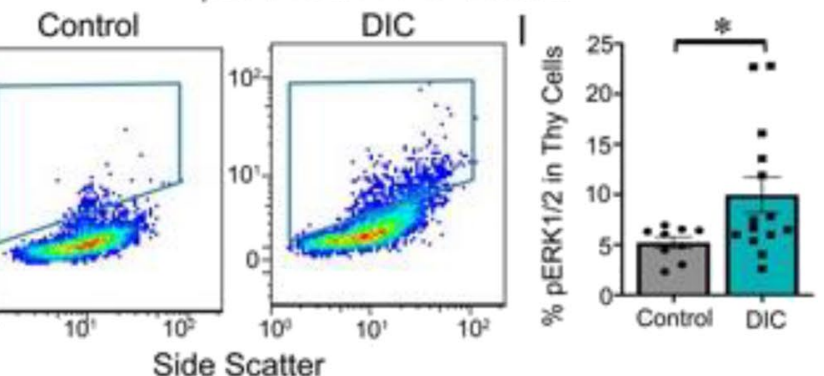

$\mathbf{g}$ and $\mathbf{h}$ and fibroblasts as assessed by ROS from mice treated with DIC compared to control mice. $n=9-13$ mice per group. $\mathbf{i}$ and $\mathbf{j}$ Increase in pERK1/2 in cardiomyocytes and $\mathbf{k}$ and $\mathbf{l}$ fibroblasts from DIC-treated and control groups. Statistical significance was considered to be achieved when $P<0.05$ by $t$-test. Representative results are shown. $X$ and $Y$ axes represent arbitrary units. Data expressed as mean \pm SEM.

three pathways by the $C O X, L O X$, and $C Y P 450$ enzymes. Since these three enzymatic pathways share the same substrates, to determine the underlying mechanism of the cardiotoxicity with chronic exposure to DIC, we analyzed the oxylipin metabolites from ARA, EPA, DHA, LA, and ALA, many of which are targeted by DIC. Sixty oxylipins were analyzed by ultrahigh-pressure LC/MS/MS from chronic DIC-treated and control plasma samples [19]. DIC-treated mice showed a significant decrease in prostanoids $\left(\mathrm{PGD}_{2}\right.$, $\mathrm{PGE}_{2}, \mathrm{PGF}_{2 \alpha}, \mathrm{TXB} 2$, 6-keto-PGF ${ }_{1 \alpha}$, and 8-iso-PGF ${ }_{1 \alpha}$ ), consistent with the COX enzymes targeting effects of DIC (Fig. 5a). Unsupervised PCA revealed a separation of samples based on DIC treatment and control groups (Fig. 5b). 

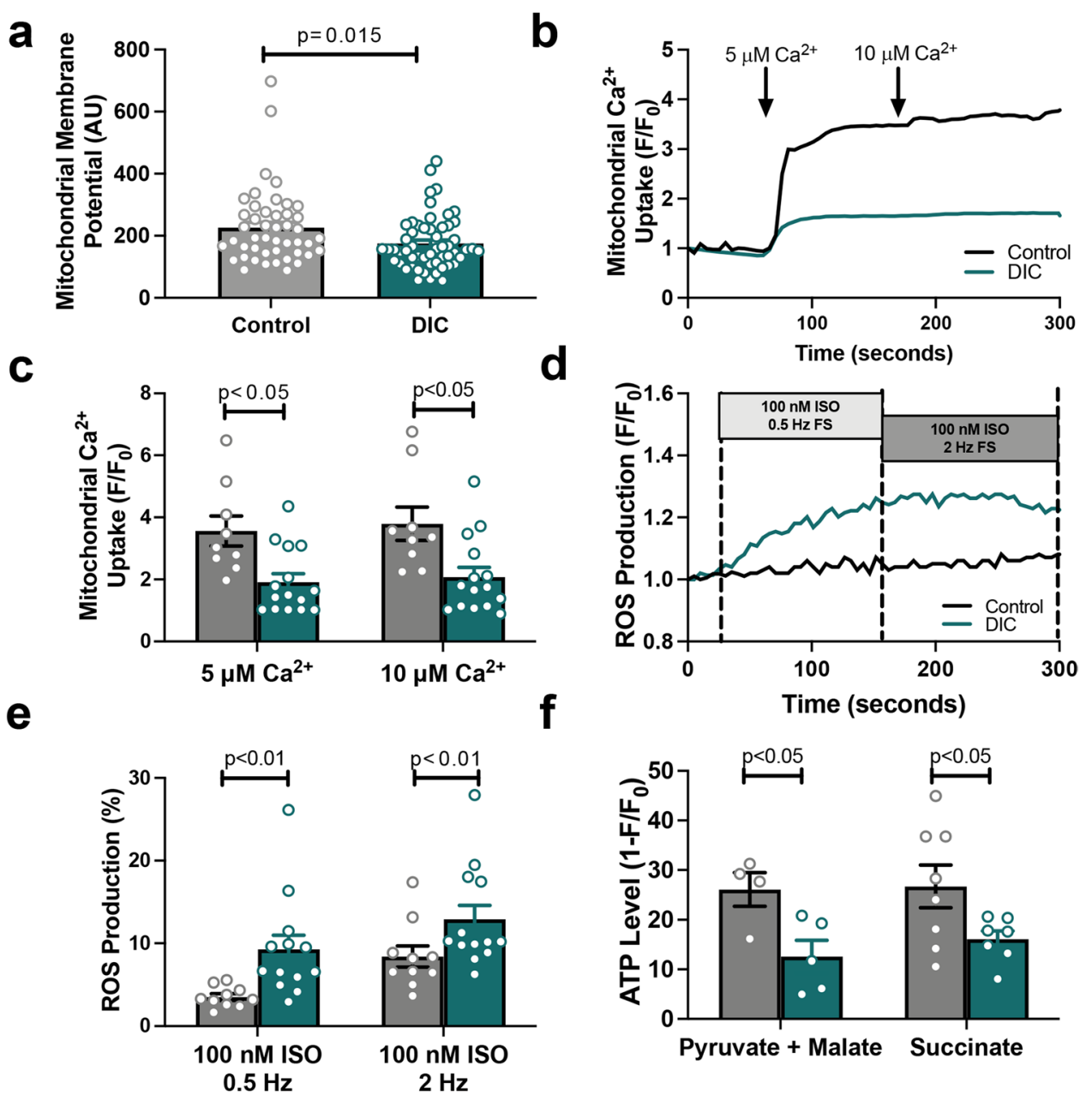

Fig. 4 Chronic DIC treatment impaired mitochondrial function. Mitochondrial functional assessment was done in isolated cardiomyocytes. a Mitochondrial membrane potential was monitored using TMRM. With DIC treatment, mitochondrial membrane potential became more depolarized, relative to control. b Representative traces show that mitochondrial $\mathrm{Ca}^{2+}$ uptake was reduced in isolated cardiomyocytes from treated mice. c Quantitatively, mitochondrial $\mathrm{Ca}^{2+}$ was significantly lower at external $\left[\mathrm{Ca}^{2+}\right]$ of $5 \mu \mathrm{M}$ and $10 \mu \mathrm{M}$ in treated cardiomyocytes. d To see the effects of ROS production from the mitochon-

Indeed, a hierarchical cluster analysis using Ward's algorithm showed clustering of metabolites from the DIC-treated group, that was separated from clustering of metabolites from the control group (Fig. 5c, green-DIC and red-control). To further explore these differences, a PLS-DA was performed. This resulted in a four-component model with a goodness of prediction $Q^{2}$ of 0.28 and a goodness of fit $R^{2}$ of 0.71 (Fig. 5d).

Of interest in cardioprotection is the metabolism of ARA by CYP450 enzymes to epoxyeicosatrienoic acidsepoxyeicosatrienoic acids (EETs), that are known to anti-inflammatory metabolites. However, EETs are further metabolized into dihydroxyeicosareinoic acid (DHETs) with diminished cardioprotective activities. Therefore, to understand the effect of dria, cardiomyocytes were stimulated with both field stimulation and isoproterenol, as shown in the representative traces. e In both conditions, ROS production was greater in treated cardiomyocytes. $\mathbf{f}$ Moreover, ATP level was significantly reduced in treated cardiomyocytes after Complex I and Complex II mediated respiration. Data expressed as mean \pm SEM. $n=3-6$ mice for each group. Statistical significance was considered to be achieved when $P<0.05$ by $t$-test and repeated measures $t$-test

DIC treatment on ARA and CYP450 pathway, we analyzed the EETs/DHETs ratio. Our analysis demonstrated a significant decrease in the 14,15-EET/DHET ratio in the DICtreated groups compared with control group (Figure S2A). Moreover, metabolites of the $L O X$ pathways demonstrated a modest difference between the two groups (Figure S2B).

While DIC suppresses inflammation, chronic DIC treatment is associated with increased levels of inflammatory cytokines including TNF- $\alpha$ [22]. Our data demonstrate a significant increase in proinflammatory cytokine and chemokine levels including interleukin 6 (IL-6), interferon- $\gamma$ (IFN- $\gamma$ ), TNF- $\alpha$, and monocyte chemoattractant protein-1 (MCP-1) in the DIC-treated mice compared to controls (Fig. 5e-h). 


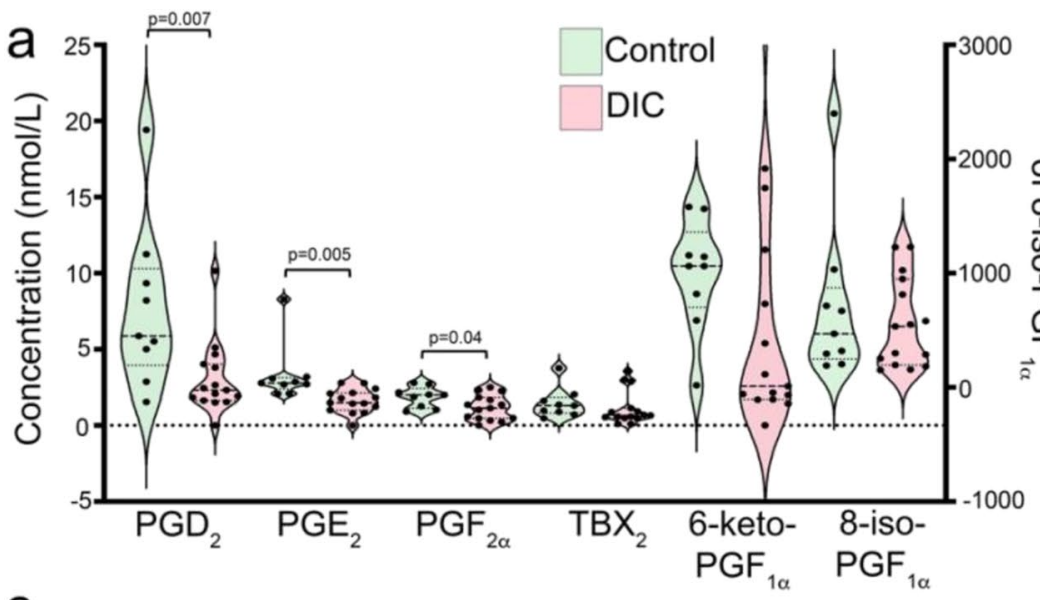

C

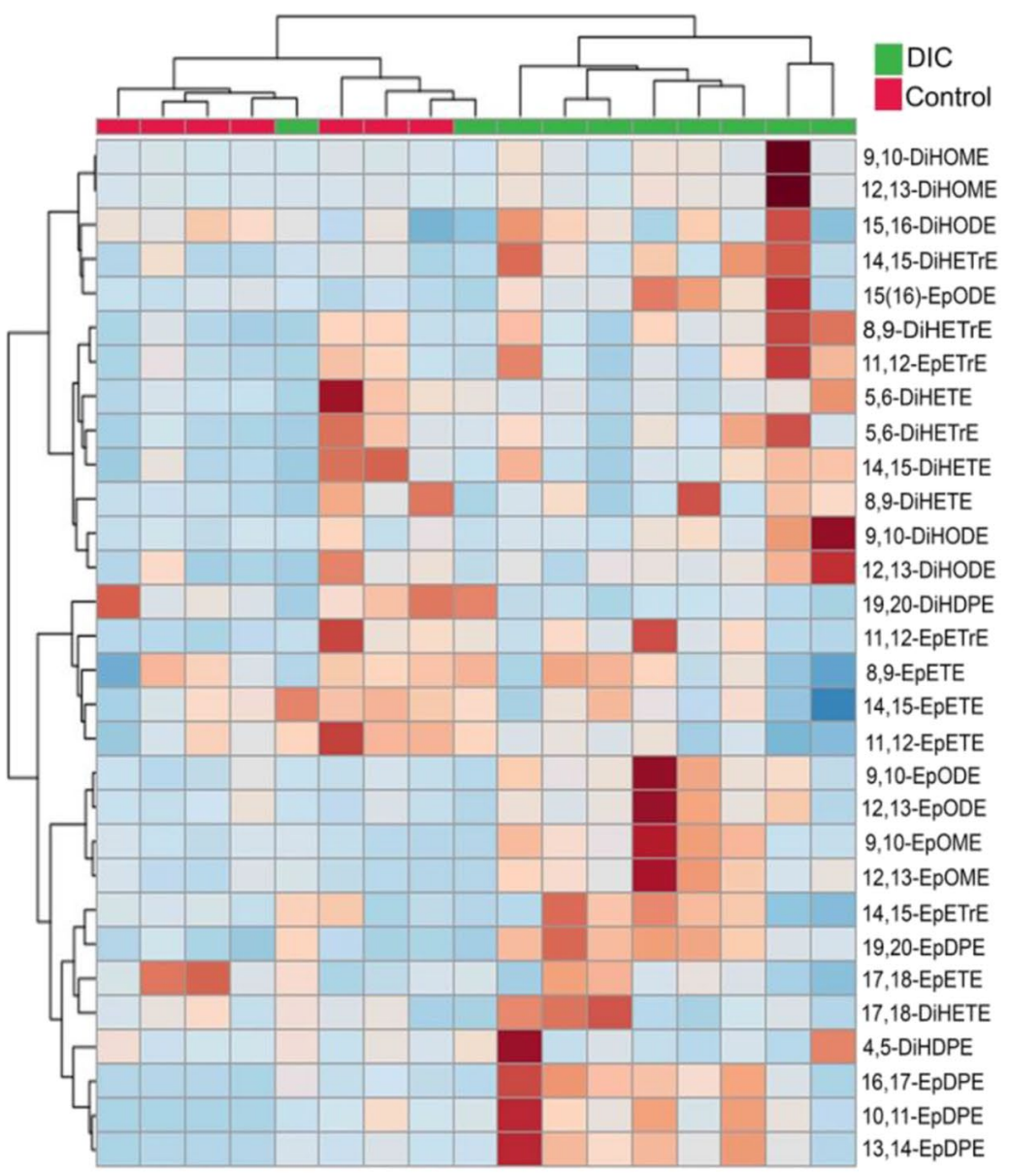

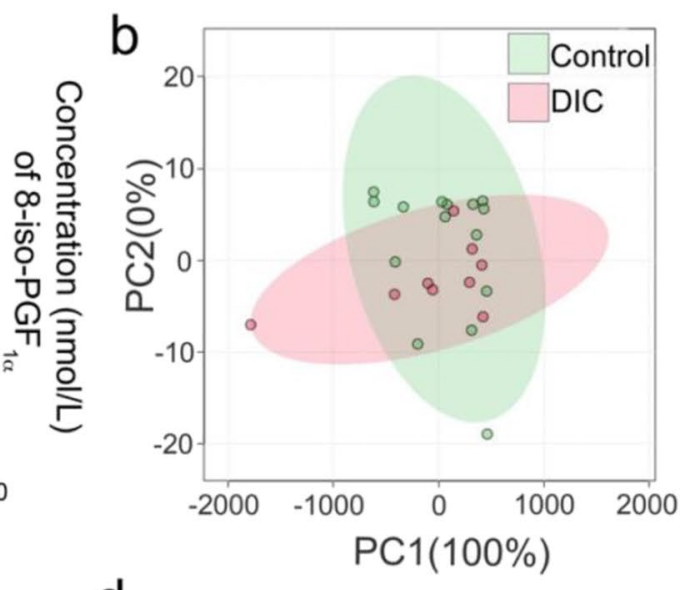

d
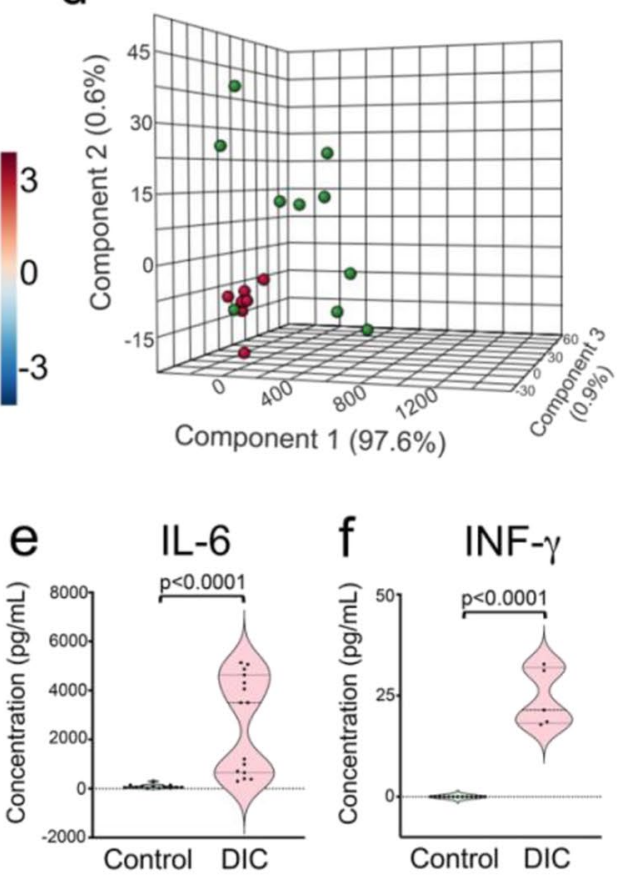

$\mathrm{g}$

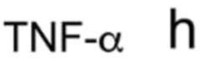

MCP-1
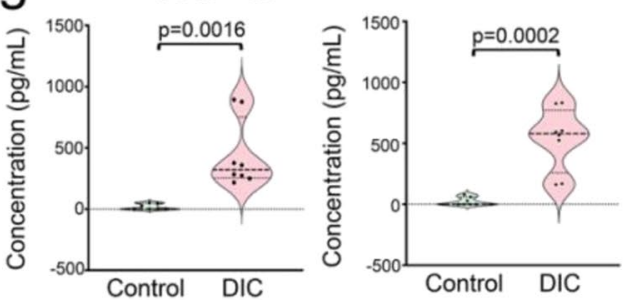

Fig. 5 Chronic DIC treatment alters the oxylipins and increases inflammatory cytokines. a Plasma oxylipin concentration of prostanoids from DIC-treated and control groups. b Principal component analysis comparing prostaglandin concentrations between DICtreated and control groups. c Heat map representing ARA, EPA, DHA, LA, and ALA metabolites post normality analysis. The color scale from -3 to 3 represents the $z$-score. A positive $z$-score (increase in red color intensity) reflects increased metabolite concentration and a negative $z$-score (increase in blue color intensity) reflects decreased concentration. Red and green colored boxes on top of the heatmap represent the two groups (red=DIC treatment and green $=$ control group) with their position showing how they cluster together, as determined by hierarchical clustering using Ward's algorithm. d Partial least-squares discriminant analyses comparing metabolites from c. e-h Serum concentration of inflammatory cytokines and chemokine. Data expressed as mean \pm SEM. $n=10-16$ mice for each group. Statistical significance was considered to be achieved when $P<0.05$ by $t$-test or one-way ANOVA with post hoc Bonferrori's test 


\section{Discussion}

The widespread availability, usage, and improper disposal of NSAIDs are not only detrimental to the environment [2], but also harmful to humans [24]. The cardiotoxic effects of NSAIDs vary among individual drug and also is dosedependent. In the current study, we demonstrate that 4 weeks of chronic exposure to DIC, a commonly prescribed NSAID, and in terms of tonnage, one of world's major medication, result in impaired cardiac systolic and diastolic function (Fig. 1), as well as increased cardiac fibroblast proliferation (Fig. 3). Although the risk of cardiovascular complications such as blood pressure elevation [24] and arrhythmias [3, 25] is higher in patients who take NSAIDs, we did not observe any differences in blood pressure or any arrhythmias in the mice that were treated relative to control mice. This is likely attributed to the regimen chosen. However, the 4-week treatment plan was chosen to investigate DIC-induced cardiotoxicity, since we observed an increase in mortality of mice with this schedule (Figure S1B). Further investigation demonstrated that chronic DIC treatment severely affected the digestive system, manifested by ischemic colitis in treated mice that did not survive the 4-week treatment regimen. The effect of NSAIDs on the gastrointestinal tract (GI) is due to the unique local environment, the pharmacokinetics of the drugs, and mitochondrial dysfunction. The effects in the GI tract may result in high mortality in mice treated with DIC and not cardiac dysfunction alone. The decreased levels of prostanoids, in particular $\mathrm{PGE}_{2}$ with $\mathrm{COX}$ inhibition, lead to removal of the protection in the GI, leading to gastric ulcers and erosion of gastric cells [31]. The accumulation of NSAIDs in the GI lining independently causes mitochondrial dysfunction, leading to GI damage [32].

\section{Diclofenac and $\mathrm{Ca}^{2+}$ Handling}

$\mathrm{Ca}^{2+}$ handling is tightly regulated for normal cardiac function. Since $\mathrm{Ca}^{2+}$ is an important regulator that mediates an array of cardiac signaling pathways [33], alterations in $\mathrm{Ca}^{2+}$ can result in severe cardiac consequences [34]. Indeed, one primary role of $\mathrm{Ca}^{2+}$ in the heart is to couple energy production with energy demand [35], which involves proper $\mathrm{Ca}^{2+}$ communication between the SR and the mitochondria at microdomains [36]. It has been reported that DIC inhibits L-type $\mathrm{Ca}^{2+}$ channels in cardiomyocytes [37], which likely affects $\mathrm{Ca}^{2+}$-induced $\mathrm{Ca}^{2+}$ release from the SR. Indeed, our in vitro findings showed isolated cardiomyocytes from treated mice exhibited impaired $\mathrm{Ca}^{2+}$ transient peak amplitude and kinetics, as well as depressed sarcoplasmic reticulum (SR) load (Fig. 2).

It has been shown that $\mathrm{Ca}^{2+}$ entry into the mitochondria is crucial for activating key dehydrogenases, which subsequently increases production of reduced NADH and $\mathrm{FADH}_{2}$ to further fuel oxidative phosphorylation [38]. Along with the reduced $\mathrm{Ca}^{2+}$ transient peak amplitude, we found depressed mitochondrial $\mathrm{Ca}^{2+}$ uptake when challenged with increasing concentrations of external $\mathrm{Ca}^{2+}$, which suggests impaired communication with the SR and mitochondria with chronic DIC treatment (Fig. 4). Additionally, our data demonstrate that chronic DIC treatment reduced ATP levels after Complex I and Complex II mediated respiration in isolated cardiomyocytes. Although a reduction in $\mathrm{Ca}^{2+}$ transient, $\mathrm{SR}$ load, and reduced ability of mitochondria to uptake $\mathrm{Ca}^{2+}$ contribute to lower energy production, ROS production can also impair mitochondrial function.

\section{Diclofenac and Oxidative Stress}

DIC has been shown to increase the production of ROS in several cell types including mouse neonatal cardiomyocytes [21]. Members of the MAPK signaling pathway induce cardiac fibrosis by activating the proliferation and collagen production of cardiac fibroblasts [39]. Specifically, an increase of ERK1/2 activity activated by increased ROS enhances cardiac fibroblast proliferation and thereby cardiac fibrosis and dysfunction $[19,20]$. In response to increased ROS, fibroblasts can produce proinflammatory chemokines and chemokines, leading to exacerbation of cardiac dysfunction. Our study shows a significant increase in ROS and a concomitant increase of activated ERK1/2 in cardiomyocytes and fibroblasts from mice treated with DIC (Fig. 3). The elevated ROS levels and the activation on ERK1/2 in our study suggest that increase in cardiac fibroblast proliferation was partly due to the activation of the MAPK signaling cascade initiated by ROS.

Furthermore, we found that mitochondrial membrane potential $(\Delta \Psi \mathrm{m})$ from DIC-treated mice was severely more depolarized than control mitochondria (Fig. 4), which suggests impaired mitochondrial function. A previous study has found that elevated levels in $\mathrm{PGE}_{2}$ can dissipate membrane potential, [40] which may partly explain our depolarized mitochondrial membrane potential. Similarly, a recent study by Barndolini et al., evaluating the effects of ketoprofen and DIC in immortalized human cardiomyocytes, demonstrates a decrease in mitochondrial potential. One possible underlying mechanism may be due to the opening of the mitochondrial permeability transition pores promoted by the $\mathrm{Ca}^{2+}$ accumulation in mitochondria. [16]

Indeed, mitochondria from DIC-treated mice were more susceptible to ROS generation under basal conditions and after $\beta$-AR stimulation and field stimulation. Mitochondrial permeability transition pores are stimulated by mitochondrial $\mathrm{Ca}^{2+}$ accumulation to facilitate the free passage of low molecular weight compounds between the inner mitochondrial matrix and the cytosol [16]. The underlying mechanism 
of DIC-dependent depolarization may in part be due to inefficient opening of the mitochondrial permeability transition pores, which is exacerbated by impaired mitochondrial $\mathrm{Ca}^{2+}$ uptake shown in our study.

It has been reported that DIC toxicity was evident in the ultrastructure of livers from chicks fed a DIC diet [41]. In addition to multiple structural alterations in the organelles, the authors noted the prevalence of diminished mitochondrial membrane integrity. These structural alterations due to DIC toxicity can lead to impaired mitochondrial function, as supported in our study. Furthermore, another study found that glutathione (GSH), a necessary component in the cellular antioxidant machinery, is able to mitigate some of DIC-induced inhibitory effects of ATP production in rat liver mitochondria [42]. In alignment with our study, we found that in addition to decreased ATP levels with DIC treatment, there was also a significant increase in ROS generation. Our data therefore strongly suggest that mitochondrial function was overall impaired due to chronic DIC treatment, in part, by an increase in oxidative stress.

\section{Important Insights into the Mechanisms Underlying Mitochondrial Dysfunction from Chronic DIC Exposure Using Metabolomic Analyses}

Polyunsaturated fatty acids including ARA, EPA, DHA, LA, and ALA can be metabolized via three broad pathways governed by $C O X, L O X$, and CYP450 enzymes to yield prostaglandins, leukotrienes, EETs, and hydroxyeicosatetraenoic acid (HETE) [43]. The imbalance arising due to COX inhibition between prostaglandins (anti-thrombogenic) and thromboxanes (thrombogenic) is one of the main causes of thrombotic events, which increases the cardiovascular risk with $C O X$ inhibition [44]. However, DIC not only inhibits the $C O X-1$ and $C O X-2$ enzymes, but also affects other pathways including $L O X$ and $C Y P 450$ leading to a decrease in the cardioprotective anti-inflammatory metabolites [44]. We have extensively shown that EETs, the CYP450 metabolites, are anti-inflammatory and cardioprotective in preventing ventricular hypertrophy, electrical remodeling, and cardiac fibrosis and reducing both atrial and ventricular arrhythmia inducibility in cardiac hypertrophy and MI models [19, $20,45]$. Our results in this study demonstrate a decrease in EETs isomers with DIC treatment (Figure S3A) suggesting a reduction in cardioprotection with DIC treatment.

HETEs (12-HETE and 15-HETE), which are metabolized through the LOX pathway, cause mitochondrial dysfunction by decreasing mitochondrial respiration and transmembrane potential [46]. An increase in HETEs (8-, 9-, 12- and 15-HETEs, Figure S3B) and a more depolarized mitochondrial potential with DIC treatment in our study suggest that DIC increases mitochondrial ROS through modifications in oxylipin levels. It has been shown that mitochondrial
ROS not only activates PGE synthase, which provides substrate for $C O X-1$ and $C O X-2$, but also increases $C O X-2$ gene expression $[47,48]$. Thus, paradoxically, DIC induces the production of $C O X$ in multiple ways; the same enzyme that DIC is used to inhibit.

\section{Mitochondrial Oxidative Stress, Inflammatory Mediators, and Cardiac Fibrosis}

Mitochondrial oxidative stress and inflammation have both been implicated in the development of cardiac dysfunction leading to chronic heart failure[49]. The mitochondrial electron transport chain is a predominant source of intracellular ROS. Mitochondrial ROS activate the release of molecules known as danger-associated molecular patterns (DAMPs), that directly trigger the production of proinflammatory cytokines through the activation of inflammasome, leading to cardiac dysfunction[50]. The critical roles of mitochondrial ROS in the induction of cardiac fibrosis and hypertrophy leading to heart failure have been demonstrated [12] In our study, we demonstrate that chronic exposure of DIC increases mitochondrial ROS production and cardiac fibroblast proliferation and activation. Future studies are needed to determine a direct causal effect of increased mitochondrial ROS production on cardiac fibrosis, leading to cardiac dysfunction by chronic DIC exposure.

\section{Conclusions}

We demonstrate that chronic treatment with DIC results in impaired cardiac function. In corroboration with this finding, our in vitro data in isolated cardiomyocytes show reduced cell shortening and impaired $\mathrm{Ca}^{2+}$ dynamics at the cellular level. With DIC treatment, mitochondrial membrane potential becomes depolarized and ATP production is diminished. Moreover, cardiomyocytes exhibit increased ROS and mitochondria from DIC-treated mice show increased susceptibility to ROS generation after application of a $\beta$-AR agonist along with field stimulation. Taken together, our data suggest that chronic DIC treatment results in elevated oxidative stress and altered oxylipin profiles towards inflammatory features, which contributed to mitochondrial dysfunction and ultimately impaired cardiac function.

Supplementary Information The online version contains supplementary material available at https://doi.org/10.1007/s10557-021-07253-4.

Acknowledgements We would like to thank Chandra Reid for her continued administrative support. The MF20 monoclonal antibody developed by Donald A. Fischman was obtained from Developmental Studies Hybridoma Bank developed under the auspices of the NICHD 
and maintained by The University of Iowa, Department of Biology, Iowa City, IA-52242.

Author Contribution PNT and PS designed the research study, conducted experiments, acquired data, analyzed data, and wrote the manuscript. LR, WX, JO, MH, CEN, VT, and JY conducted experiments, acquired data, and analyzed data. $\mathrm{AG}$ and $\mathrm{BDH}$ provided guidance. $\mathrm{NC}$ designed the research study, provided financial support, and wrote the manuscript.

Funding This work was supported by Postdoctoral Fellowships from National Institutes of Health (NIH) T32 Training Grant in Basic \& Translational Cardiovascular Science HL86350 and NIH F32 HL149288 (PNT); AHA Predoctoral Award 18PRE34030199 (LR); NIEHS Superfund Basic Research P42ES04699 and RIVER R35 ES030443 (BDH), NIH R01 HL085727, HL085844, HL137228, and S10 RR033106, Rosenfeld Foundation Research Award, VA Merit Review Grant I01 BX000576 and I01 CX001490 (NC); Postdoctoral Fellowship from NIH T32 Training Grant in Basic \& Translational Cardiovascular Science (T32 HL86350), American Heart Association Career Development Award (18CDA34110060), and Harold S. Geneen Charitable Trust Award (PS).

Data Availability All data generated or analyzed during this study are included in this published article and its supplementary information files.

Code Availability Commercially available GraphPad Prism version 9 (San Diego, CA) and online MetaboAnalyst version 5.0 were used.

\section{Declarations}

Ethics Approval All animal work was performed in accordance with approved protocols of the Institutional Animal Care and Use (IACUC) Committee at the University of California, Davis, which adheres to the guidelines published by the US National Institutes of Health $\left(8^{\text {th }}\right.$ Edition).

Consent to Participate Not applicable.

Consent to Publish Not applicable.

Conflict of Interest The authors declare no competing interests.

Research Involving Human Subjects Not applicable.

Informed Consent Not applicable.

Open Access This article is licensed under a Creative Commons Attribution 4.0 International License, which permits use, sharing, adaptation, distribution and reproduction in any medium or format, as long as you give appropriate credit to the original author(s) and the source, provide a link to the Creative Commons licence, and indicate if changes were made. The images or other third party material in this article are included in the article's Creative Commons licence, unless indicated otherwise in a credit line to the material. If material is not included in the article's Creative Commons licence and your intended use is not permitted by statutory regulation or exceeds the permitted use, you will need to obtain permission directly from the copyright holder. To view a copy of this licence, visit http://creativecommons.org/licenses/by/4.0/.

\section{References}

1. Wongrakpanich S, Wongrakpanich A, Melhado K, Rangaswami J. A comprehensive review of non-steroidal anti-inflammatory drug use in the elderly. Aging Dis. 2018;9(1):143-50.

2. Gonzalez-Rey M, Bebianno MJ. Effects of non-steroidal antiinflammatory drug (NSAID) diclofenac exposure in mussel Mytilus galloprovincialis. Aquat Toxicol. 2014;148:221-30.

3. Schmidt M, Christiansen CF, Mehnert F, Rothman KJ, Sørensen HT. Non-steroidal anti-inflammatory drug use and risk of atrial fibrillation or flutter: population based case-control study. BMJ. 2011;343:d3450.

4. Kaufman DW, Kelly JP, Battista DR, Malone MK, Weinstein RB, Shiffman S. Exceeding the daily dosing limit of nonsteroidal antiinflammatory drugs among ibuprofen users. Pharmacoepidemiol Drug Saf. 2018;27(3):322-31.

5. Bally M, Dendukuri N, Rich B, Nadeau L, Helin-Salmivaara A, Garbe E, et al. Risk of acute myocardial infarction with NSAIDs in real world use: bayesian meta-analysis of individual patient data. BMJ. 2017;357:j1909.

6. Vane JR, Botting RM. Mechanism of action of nonsteroidal antiinflammatory drugs. Am J Med. 1998;104(3a):2S-8S (discussion 21S-2S).

7. Hanna VS, Hafez EAA. Synopsis of arachidonic acid metabolism: a review. J Adv Res. 2018;11:23-32.

8. Vane SJ. Differential inhibition of cyclooxygenase isoforms: an explanation of the action of NSAIDs. J Clin Rheumatol. 1998;4(5 Suppl):s3-10.

9. Ryan EP, Rahman I, Phipps RP. Cyclooxygenase-2 inhibition induces oxidative stress and decreases intracellular glutathione to reduce survival of human B lymphoma/leukemia cells. J Clin Oncol. 2007;25(18_suppl):18522.

10. Chen Y-R, Zweier JL. Cardiac mitochondria and reactive oxygen species generation. Circ Res. 2014;114(3):524-37.

11. Panth N, Paudel KR, Parajuli K. Reactive oxygen species: a key hallmark of cardiovascular disease. Adv Med. 2016;2016:9152732.

12. Dai DF, Johnson SC, Villarin JJ, Chin MT, Nieves-Cintrón M, Chen T, et al. Mitochondrial oxidative stress mediates angiotensin II-induced cardiac hypertrophy and Galphaq overexpressioninduced heart failure. Circ Res. 2011;108(7):837-46.

13. Brown DA, Perry JB, Allen ME, Sabbah HN, Stauffer BL, Shaikh $\mathrm{SR}$, et al. Mitochondrial function as a therapeutic target in heart failure. Nat Rev Cardiol. 2017;14(4):238-50.

14. Dhalla NS, Temsah RM, Netticadan T. Role of oxidative stress in cardiovascular diseases. J Hypertens. 2000;18(6):655-73.

15. Varga Z, Sabzwari SRA, Vargova V. Cardiovascular risk of nonsteroidal anti-inflammatory drugs: an under-recognized public health issue. Cureus. 2017;9(4):e1144.

16. Brandolini L, Antonosante A, Giorgio C, Bagnasco M, d'Angelo $\mathrm{M}$, Castelli V, et al. NSAIDs-dependent adaption of the mitochondria-proteasome system in immortalized human cardiomyocytes. Sci Rep. 2020;10(1):18337.

17. Thai PN, Daugherty DJ, Frederich BJ, Lu X, Deng W, Bers DM, et al. Cardiac-specific conditional knockout of the 18-kDa mitochondrial translocator protein protects from pressure overload induced heart failure. Sci Rep. 2018;8(1):16213.

18. Sirish P, Ledford HA, Timofeyev V, Thai PN, Ren L, Kim HJ, et al. Action potential shortening and impairment of cardiac function by ablation of Slc26a6. Circ Arrhythm Electrophysiol. 2017;10(10):e005267.

19. Sirish P, Li N, Liu JY, Lee KS, Hwang SH, Qiu H, et al. Unique mechanistic insights into the beneficial effects of soluble epoxide hydrolase inhibitors in the prevention of cardiac fibrosis. Proc Natl Acad Sci U S A. 2013;110(14):5618-23. 
20. Sirish P, Li N, Timofeyev V, Zhang XD, Wang L, Yang J, et al. Molecular mechanisms and new treatment paradigm for atrial fibrillation. Circ Arrhythm Electrophysiol. 2016 ;9(5) CIRCEP.115.003721e003721.

21. Ghosh R, Goswami SK, Feitoza L, Hammock B, Gomes AV. Diclofenac induces proteasome and mitochondrial dysfunction in murine cardiomyocytes and hearts. Int J Cardiol. 2016;223:923-35.

22. Goswami SK, Rand AA, Wan D, Yang J, Inceoglu B, Thomas M, et al. Pharmacological inhibition of soluble epoxide hydrolase or genetic deletion reduces diclofenac-induced gastric ulcers. Life Sci. 2017;180:114-22.

23. Roth DM, Swaney JS, Dalton ND, Gilpin EA, Ross J Jr. Impact of anesthesia on cardiac function during echocardiography in mice. Am J Physiol Heart Circ Physiol. 2002;282(6):H2134-40.

24. Johnson AG. NSAIDs and increased blood pressure. What is the clinical significance? Drug Saf. 1997;17(5):277-89.

25. Granier M, Massin F, Pasquié JL. Pro- and anti-arrhythmic effects of anti-inflammatory drugs. Antiinflamm Antiallergy Agents Med Chem. 2013;12(1):83-93.

26. Luo M, Anderson ME. Mechanisms of altered $\mathrm{Ca}^{2+}$ handling in heart failure. Circ Res. 2013;113(6):690-708.

27. Schmidt M, Sørensen HT, Pedersen L. Diclofenac use and cardiovascular risks: series of nationwide cohort studies. BMJ. 2018;362:k3426.

28. Eisner V, Csordás G, Hajnóczky G. Interactions between sarcoendoplasmic reticulum and mitochondria in cardiac and skeletal muscle - pivotal roles in $\mathrm{Ca}<$ sup $>2+</$ sup $>$ and reactive oxygen species signaling. J Cell Sci. 2013;126(14):2965.

29. Corbi G, Conti V, Russomanno G, Longobardi G, Furgi G, Filippelli A, et al. Adrenergic signaling and oxidative stress: a role for sirtuins? Front Physiol. 2013;4:324.

30. Görlach A, Bertram K, Hudecova S, Krizanova O. Calcium and ROS: A mutual interplay. Redox Biol. 2015;6:260-71.

31. Tonolini M. Acute nonsteroidal anti-inflammatory drug-induced colitis. J Emerg Trauma Shock. 2013;6(4):301-3.

32. Krause MM, Brand MD, Krauss S, Meisel C, Vergin H, Burmester GR, et al. Nonsteroidal antiinflammatory drugs and a selective cyclooxygenase 2 inhibitor uncouple mitochondria in intact cells. Arthritis Rheum. 2003;48(5):1438-44.

33. Fearnley CJ, Roderick HL, Bootman MD. Calcium signaling in cardiac myocytes. Cold Spring Harb Perspect Biol. 2011;3(11):a004242.

34. Yano M, Ikeda Y, Matsuzaki M. Altered intracellular Ca2+ handling in heart failure. J Clin Investig. 2005;115(3):556-64.

35. Tarasov AI, Griffiths EJ, Rutter GA. Regulation of ATP production by mitochondrial $\mathrm{Ca}(2+)$. Cell Calcium. 2012;52(1):28-35.

36. de Brito OM, Scorrano L. Mitofusin 2 tethers endoplasmic reticulum to mitochondria. Nature. 2008;456(7222):605-10.

37. Yarishkin OV, Hwang EM, Kim D, Yoo JC, Kang SS, Kim DR, et al. Diclofenac, a non-steroidal anti-inflammatory drug, inhibits L-type Ca channels in neonatal rat ventricular cardiomyocytes. Korean J Physiol Pharmacol. 2009;13(6):437-42.

38. Denton RM. Regulation of mitochondrial dehydrogenases by calcium ions. Biochim Biophys Acta. 2009;1787(11):1309-16.
39. Pan Z, Zhao W, Zhang X, Wang B, Wang J, Sun X, et al. Scutellarin alleviates interstitial fibrosis and cardiac dysfunction of infarct rats by inhibiting TGFbeta1 expression and activation of p38MAPK and ERK1/2. Br J Pharmacol. 2011;162(3):688-700.

40. Sanin DE, Matsushita M, Klein Geltink RI, Grzes KM, van Teijlingen BN, Corrado M, et al. Mitochondrial membrane potential regulates nuclear gene expression in macrophages exposed to prostaglandin E2. Immunity. 2018;49(6):1021-33.e6.

41. Haritha C, Gopala RA, Anjaneyulu Y, Kalakumar B, Dilip RG. Oxidative stress induced by diclofenac alone and under the influence of certain variables in broilers. Toxicol Int. 2010;17(1):27-9.

42. Syed M, Skonberg C, Hansen SH. Mitochondrial toxicity of diclofenac and its metabolites via inhibition of oxidative phosphorylation (ATP synthesis) in rat liver mitochondria: possible role in drug induced liver injury (DILI). Toxicol In Vitro. 2016;31:93-102.

43. Li N, Liu JY, Qiu H, Harris TR, Sirish P, Hammock BD, et al. Use of metabolomic profiling in the study of arachidonic acid metabolism in cardiovascular disease. Congest Heart Fail. 2011;17(1):42-6.

44. Ahmed SA, Al-Lawati H, Jamali F. Dose-dependency of the cardiovascular risks of non-steroidal anti-inflammatory drugs. Inflammopharmacology. 2019;27(5):903-10.

45. Sirish P, Thai PN, Lee JH, Yang J, Zhang XD, Ren L, et al. Suppression of inflammation and fibrosis using soluble epoxide hydrolase inhibitors enhances cardiac stem cell-based therapy. Stem Cells Transl Med. 2020;9(12):1570-1584.

46. Li Q, Mao M, Qiu Y, Liu G, Sheng T, Yu X, et al. Key role of ROS in the process of 15-lipoxygenase/15-hydroxyeicosatetraenoiccidinduced pulmonary vascular remodeling in hypoxia pulmonary hypertension. PloS One. 2016;11(2):e0149164.

47. Kiritoshi S, Nishikawa T, Sonoda K, Kukidome D, Senokuchi $\mathrm{T}$, Matsuo T, et al. Reactive oxygen species from mitochondria induce cyclooxygenase- 2 gene expression in human mesangial cells: potential role in diabetic nephropathy. Diabetes. 2003;52(10):2570-7.

48. Zhuang Y, Wang C, Wu C, Ding D, Zhao F, Hu C, et al. Mitochondrial oxidative stress activates COX-2/mPGES-1/PGE2 cascade induced by albumin in renal proximal tubular cells. Oncotarget. 2018;9(10):9235-45.

49. Peoples JN, Saraf A, Ghazal N, Pham TT, Kwong JQ. Mitochondrial dysfunction and oxidative stress in heart disease. Exp Mol Med. 2019;51(12):1-13.

50. Chen G, Chelu MG, Dobrev D, Li N. Cardiomyocyte inflammasome signaling in cardiomyopathies and atrial fibrillation: mechanisms and potential therapeutic implications. Front Physiol. 2018;9:1115.

Publisher's Note Springer Nature remains neutral with regard to jurisdictional claims in published maps and institutional affiliations. 